15

ournal of

Mechanics of

Materials and Structures

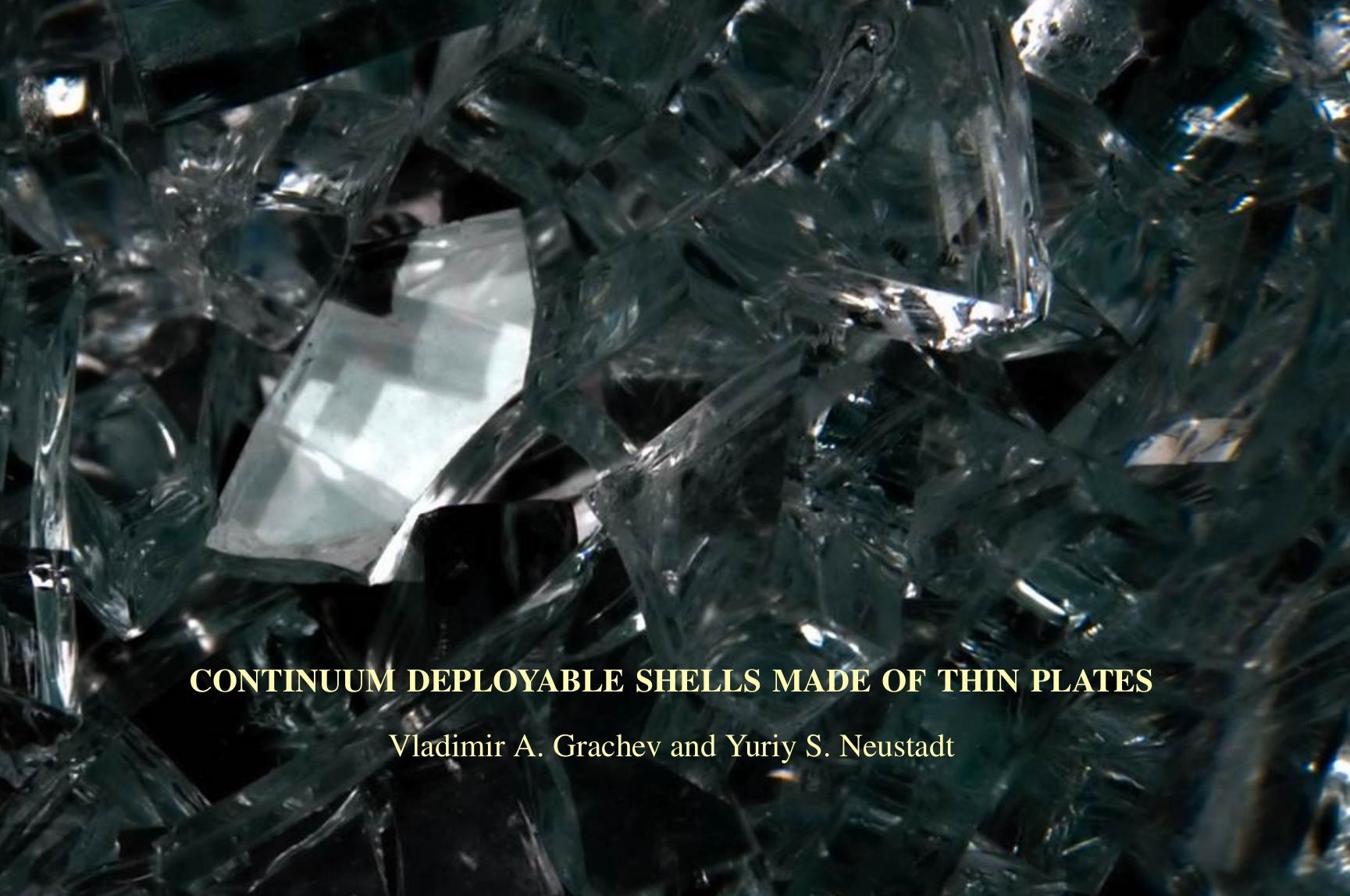

Volume- 8 ,

Volume 8, No. 2-4 \& April-June 2013

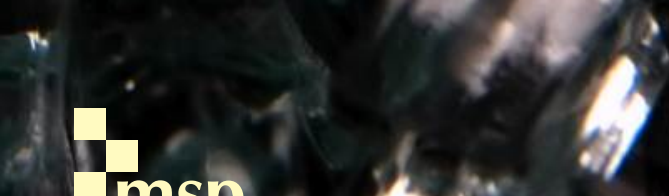




\title{
CONTINUUM DEPLOYABLE SHELLS MADE OF THIN PLATES
}

\author{
Vladimir A. GracheV AND Yuriy S. NEUSTADT
}

\begin{abstract}
This paper investigates deployable systems assembled from trapezoidal plates. When the plate package is unwrapped, a net shell with six loop cells is formed. Additional degrees of freedom appear when correlations are present between the sizes of the six loop faces. When thin plates are used, the continuum approximation of the deployed net can be interpreted as a shell with a wide variety of local curvatures. The kinematics of the continuum model are analyzed using the Cartan moving hedron method. Several practical applications of the continuum nets are demonstrated.
\end{abstract}

\section{Introduction}

A great deal of interest has been directed to the subject of deployable (transformed) mechanical systems over the past several decades. Deployable mechanisms can be easily "packed into a small box" and can create a surface with a large area after deployment [Gantes 2001; Jensen and Pellegrino 2002; Gan and Pellegrino 2006; Leung and Guest 2007; Chen and You 2008a; 2008b]. With a variety of designs, these systems have numerous potential engineering applications.

Transformed systems have one specific feature: all known stable deployable systems are standard machines; that is, they are a set of absolute solids connected with cylindrical and spherical hinges. The hinges are "internal degrees of freedom" that make controlled transformation of the mechanism possible. Thus, the design of the links does not matter for deployment (kinematics) of the relevant mechanism. The elements of the system could be given any rigidity, and the required internal degrees of freedom could be achieved using a sufficient number of hinges. Until recently, new forms of deployable systems have been limited to mechanisms with rigid parts and few degrees of freedom [Vepa 2010; Sclater and Chironis 2011].

Basically, standard machines are prestressed systems [Otto and Rasch 1995; Luchsinger et al. 2004] because the latter can be interpreted as continuum sets consisting of infinitely small rigid members (frameworks or shells) connected with spherical hinges. Such systems have numerous degrees of freedom and can sustainably move (or balance) at prestressed and special loads. This is why it would be an interesting challenge to make machines with additional degrees of freedom by replacing absolutely rigid parts with flexible ones. At the same time the number of degrees of freedom should not be large, in order to ensure sustainable control over the structure. State-of-the-art technologies allow the making of such machines.

When elastic and flexible thin plates were used as loops that could twist, the system had additional degrees of freedom that enabled a nonstandard mechanism to be deployed. In this case, a minimum amount of energy was required for a system with a high bearing capacity. The current paper describes a

Keywords: continuum deployable shells, manifold, Cartan moving hedron, homogenization, Gauss-Codazzi equations, practical application. 
similar type of mechanism, and the systems presented in this paper consist of 3D six-link loops that are not simply mechanisms arbitrarily correlated between their sizes. For this reason, the six-link loops with internal degrees of freedom are extracted first. Next, a continuum model (subsequently referred to as the continuum net or continuum shell) that approximates the finite-dimensional net of the six-link loops is constructed. The mechanical behavior of the continuum nets is studied, assuming that the cylindrical hinges between the plates are ideal and have no friction.

The paper contains an illustration of possible applications of continuum nets in engineering.

\section{Kinematics of deployable shells assembled from trapezoidal plates}

If a net constructed by sticking several paper six-link loops together consists of equal rectangular plates (shown in Figure 1, Sketch 2), unwrapping the package would cause the middle lines of the plates to form a plane. The same result would be observed if rectilinear strips of hinged rectangles were used instead of six-link loops (Figure 1, Sketch 1).

The net represents a mechanism with sides of arbitrary sizes, regardless of the number of plates it includes. The hinged hexagon has six degrees of freedom on the plane, while each glued face removes three degrees of freedom.
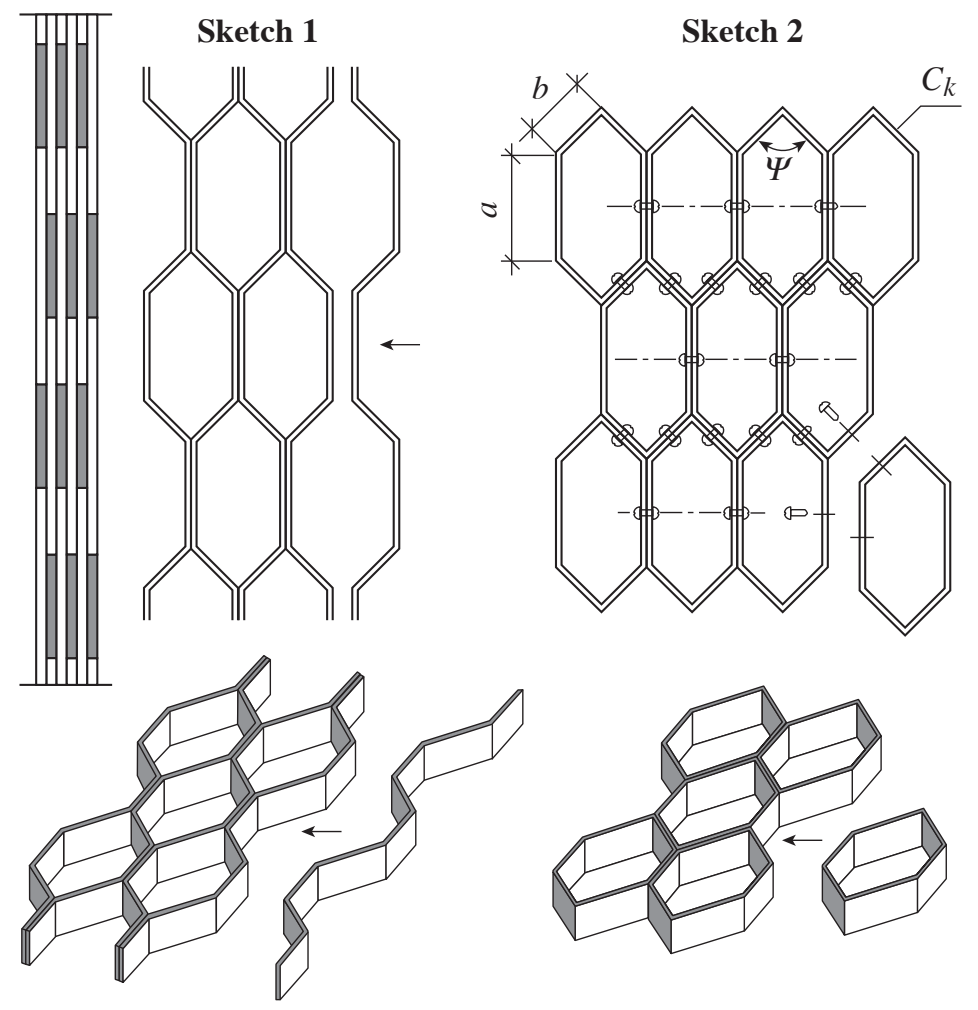

Figure 1. Net of rectangles connected with cylindrical hinges. 


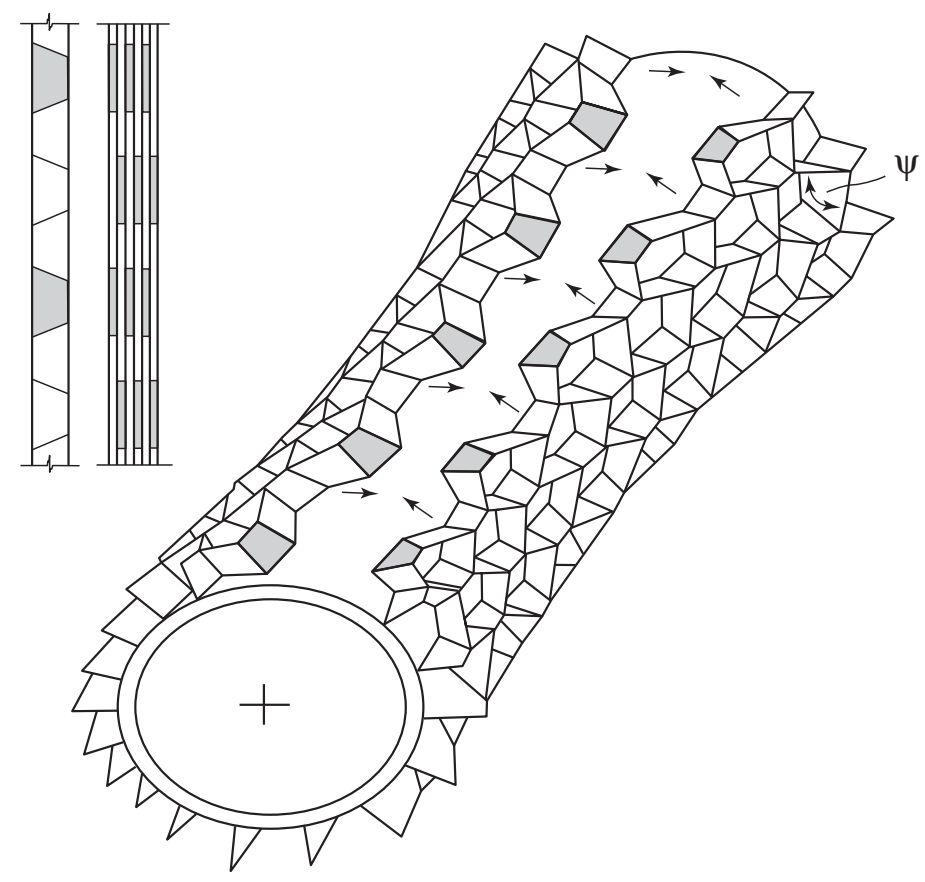

Figure 2. Net of strips assembled from trapezoids.

If undistorted rectangles in the cell were replaced with trapezoids using cylindrical hinges as a connection, the assembled net would not deploy because a six-link loop of absolute solids is usually a rigid body, while a net of $N$ six-link loops (as shown in Figure 1, Sketch 2) has $12 N$ redundant constraints.

Assuming that the cell is symmetrical (the cell consists of two part types) and the plates are thin (their rigidity for twist and bending is small), the package can be deployed into a shell (Figure 2).

The present structure was invented by Swank [1991] (in the USA) and Grachev and Neustadt [1999a] (in Russia). The research of the latter [Grachev and Neustadt 1995; 1996] covers the mechanical fundamentals of deployable systems assembled from identical trapezoidal plates. The concepts of the abovementioned articles and [Grachev and Neustadt 1999a; 1999b] are represented in a general geometrical context that enables larger classes of transformed shells to be reviewed from a common standpoint.

Making a net of trapezoidal plates according to Figure 1, Sketch 1, is more technically feasible; however, it is more convenient to construct theoretical designs according to Figure 1, Sketch 2. Therefore, the second option is used in the subsequent presentation.

Let us consider the mirror-symmetrical six-link loop that is symmetrical against two planes and is assembled from two types of trapezoids (light and dark) connected with cylindrical hinges (Figure 3). The black points are located on the middle lines of the trapezoids. Point $O$ is the center of gravity of trapezoids 2 and 5. Vector $\vec{\beta}_{1}$ is tangential to the circumference passing through the intersection of the middles lines of trapezoids 2 and 5 , and vector $\vec{\beta}_{2}$ is tangential to the arc of the circle passing through points $4, O$, and 1 .

First, it must be confirmed that the mirror-symmetrical six-link loop is a mechanism with one degree of freedom, and the surface built on the middle lines of the trapezoids serves as an approximation of 


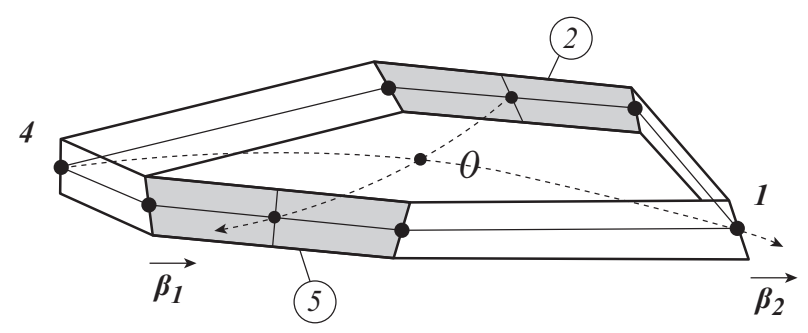

Figure 3. Six-link loop cell.

the smooth shell. For this reason, the cell shown with relevant projections in Figure 4 is considered. The values in the circles refer to the numbers of the trapezoids that comprise the hinged six-link loop. Intersections of the trapezoid centerlines are denoted with Arabic numerals.

The same values describe a linear hinge between the faces. The radius vectors of the middle lines are designated by $\vec{r}_{i}, i=1, \ldots 6$, while vector $\vec{r}_{0}$ connects the center of gravity of the cell with point 1 (Figure 5).

The angular velocity vector of the side number $i$ relative to the side $(i+1)$ is designated with $\vec{\omega}_{i}$, while the vectors of dihedral angles between the neighboring plates are designated by $\vec{\Psi}_{i}, i=1,2, \ldots, 6$. The length of the middle line of sides $1,3,4$, and 6 is equal to $a$, while the length of sides 2 and 5 is equal to $b$. The thickness of all plates is considered to be the same and equal to $t$. Cartesian $X$ and $Y$ axes are located along the plane of the vectors $\vec{r}_{2}$, whereas $\vec{r}_{0}$ and the $Z$ axis are perpendicular to the $X$ and $Y$ axes, respectively.
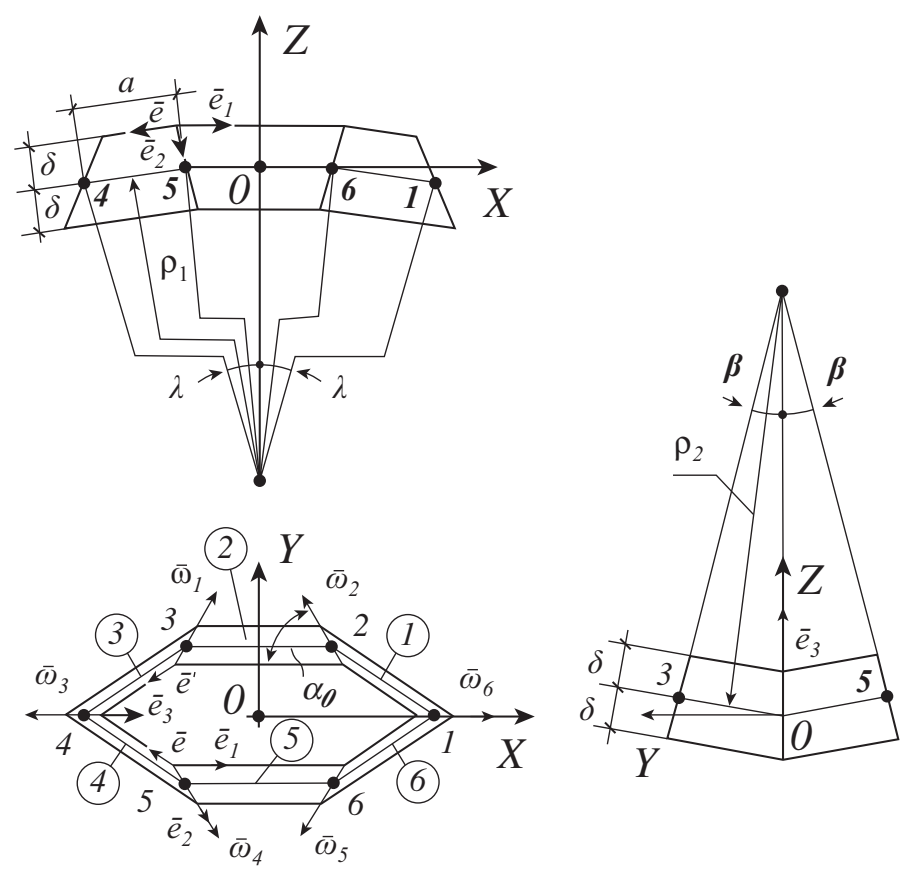

Figure 4. Sizes of the six-link loop cell. 


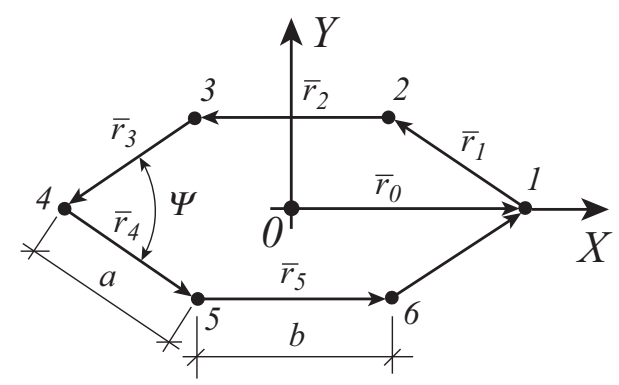

Figure 5. Middle lines of the trapezoid within the cell.

It should be noted that if the sides of the six-link loop are absolutely rigid plates connected with linear cylindrical hinges, the structure is not actually a mechanism. In fact, the number of degrees of freedom in the six-link loop is equal to

$$
F=6 \times 6-6 \times 5=6
$$

because each rigid plate has six degrees of freedom, and the linear hinge subtracts five degrees of freedom. Thus, the arbitrarily scaled six-link loop can move through space only as an absolute solid. For additional degrees of freedom to appear, the mechanism should be specially designed. The symmetrical six-link loop needs at least one degree of freedom, which could be angle $\Psi$ (Figure 4).

Limitations are set on the six angular velocities $\vec{\omega}_{i}$ [Lurye 1961] in this study. The closure of the polyhedron and the theorem of composition of angular velocities give

$$
\sum_{i=1}^{i=6} \vec{\omega}_{i}=0 .
$$

The three equations (1) are supplemented with another triple representing the fact that the velocity of the point located at the intersection of the sides is identical regardless of the side upon which it is calculated: $\vec{\omega}_{1} \times \vec{r}_{1}+\left(\vec{\omega}_{1}+\vec{\omega}_{2}\right) \times \vec{r}_{2}+\left(\vec{\omega}_{1}+\vec{\omega}_{2}+\vec{\omega}_{3}\right) \times \vec{r}_{3}+\left(\vec{\omega}_{1}+\vec{\omega}_{2}+\vec{\omega}_{3}+\vec{\omega}_{4}\right) \times \vec{r}_{4}$

$$
+\left(\vec{\omega}_{1}+\vec{\omega}_{2}+\vec{\omega}_{3}+\vec{\omega}_{4}+\vec{\omega}_{5}\right) \times \vec{r}_{5}+\left(\vec{\omega}_{1}+\vec{\omega}_{2}+\vec{\omega}_{3}+\vec{\omega}_{4}+\vec{\omega}_{5}+\vec{\omega}_{6}\right) \times \vec{r}_{6}=0 .
$$

The last equality with

$$
\sum_{i=1}^{i=6} \vec{r}_{i}=0
$$

implies that the sum of $\vec{\omega}_{i}$ vector moments with respect to point $O$ is equal to zero. When $\vec{r}_{i}$ is constant, (1) and (2) prove that there is always a trivial solution

$$
\vec{\omega}_{i}=0
$$

when the six-link loop remains geometrically unchanged. However, nontrivial solutions exist with additional limitations. It will be demonstrated that (1) and (2) allow for a nontrivial solution for a cell that is symmetrical with respect to the $X$ axis. 
Assuming $\vec{\omega}_{1}, \vec{\omega}_{2}, \vec{\omega}_{3}$, and $\vec{\omega}_{6}$ are unknown, the $Y$-axis projection equation and two moment equations with respect to the $X$ and $Y$ axes can be identically satisfied by symmetry of vectors. Thus, only three independent equations exist for four unknown vectors. Therefore, a symmetrical cell is a mechanism with one degree of freedom, due to

$$
\frac{d \vec{\Psi}_{i}}{d t}=\vec{\omega}_{i}
$$

and the symmetrical configuration at the initial instant.

This conclusion can be reached if a three-link loop consisting of plates 1, 2, and 3 is considered (the plates generally have arbitrary sizes), and the initial position on the $X O Z$ axis (Figure 4) is located on the fixed axis 1 . The position of axis 4 is a function of the three dihedral angles $\vec{\Psi}_{1} / 2, \vec{\Psi}_{2}$, and $\vec{\Psi}_{3}$.

If the angle $\vec{\Psi}_{1}$ is determined arbitrarily, the other two can be determined such that axis 4 is on the $X O Z$ plane. Flipping the three-link loop against this plane proves that the six-link loop is a system with one degree of freedom.

The same result can be obtained if a cell has two axes of symmetry. In this case, $\left|\vec{\omega}_{3}\right|=\left|\vec{\omega}_{6}\right|$, and $\left|\vec{\omega}_{1}\right|=\left|\vec{\omega}_{2}\right|$. Only one equation (the equation for the angular velocity of the $Z$-axis projection) out of six for two unknown vectors is not identically satisfied. The angle $\Psi$ is chosen as the only generalized coordinate of the cell's symmetrical deformation (Figure 4). With this angle, all angles of the cell can be calculated. The calculated cells will be designated by $\Psi_{i}, i=1,2, \ldots, 6$, for $\Psi_{4}=\Psi$. The dihedral angle between plates 3 and $4\left|\vec{\Psi}_{1}\right|=\Psi$ (Figure 4) is considered the only generalized coordinate of the cell with symmetrical distortion. Knowing this angle, all of the other dihedral angles of the cell can be calculated.

The set of cells can be connected according to Figure 1, Sketch 2, by gluing trapezoids of the same size. This operation is feasible when $\Psi=0$. If the plates are thin, and the sizes of the six-link loops are small compared to the shell dimensions (the net contains a large number of mirror-symmetrical cells), then the strip package can be unwrapped into a two-dimensional surface almost without applying any external forces.

Because the plates comprising the net are thin (the $t / \delta$ ratio is small), only a small amount of force is required to twist and bend them. Thus, the twist angles of the plates to a first approximation can be considered to be additional degrees of freedom of the lattice system.

The size of the honeycombs is assumed to be small. This assumption is equivalent to the distances between close points regarded as differentials of the relevant functions. Therefore, it is possible to parameterize and homogenize a system that contains a large number of honeycombs.

Let us connect $2 n$ strips from the trapezoids (the strips are numbered with Arabic numerals) at the initial instant shown in Figure 1, Sketch 1 (gluing even and odd strips), and unwrap the package (refer to Figure 6).

Broken lines connecting the middle lines of the odd strips (shown with a dotted line) are termed coordinate lines $\alpha_{1}$. Similarly, the lines $\alpha_{2}$ are drawn through the centers of the glued trapezoids. The Lagrangian coordinate lines $\alpha_{1}$ are considered equal to the actual length along the middle lines of the trapezoids measured from the fixed point $B$. When moving to the neighboring cell, the change in the coordinate $\alpha_{2}$ is equal to $2 b$. The discrete coordinate net formed in this manner is supplemented with continuous points of two-dimensional manifolds. 


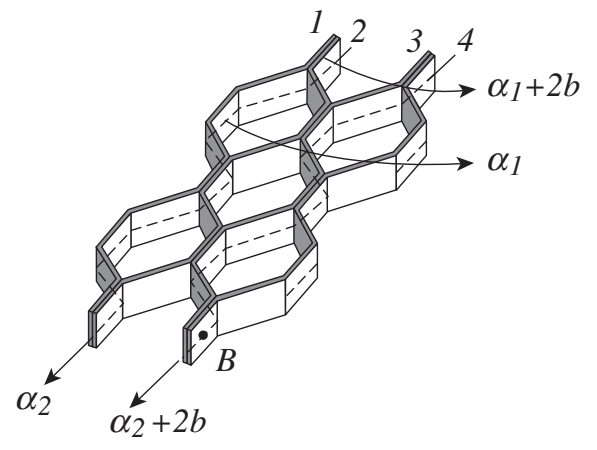

Figure 6. Lagrangian coordinates of the shell.

If the coordinate lines are closely spaced, it is possible to linearly approximate the joint coordinates of the net that are located on the middle lines of the trapezoids across the entire two-dimensional manifold; the first and second quadratic forms of the built continuum surface can also be calculated. The approximation results in a surface, a small section of which is shown in Figure 7 . The lines of the main curvature are designated by $\beta_{1}$ and $\beta_{2}$. The tangent plane passing through the center of gravity of the middle cell $O$ is designated $\Pi$.

The map consisting of projections of the middle lines of the five cells on plane $\Pi$ (Figure 7$)$ has $3 \times(8-$ $6)=6$ degrees of freedom in space (assuming that it is possible to rotate the osculating trapezoids against the middle lines). Because all cells are mirror-symmetrical, the map (Figure 7) can be supplemented with two more cells (marked as 6 and 7 in circles in Figure 8) such that the seven cell units have six degrees of freedom in space.

The middle line between points 2 and 3 of cell 6 coincides with the relevant line of the middle cell and ensures two degrees of freedom in the entire system. This number is sufficient to combine the line between points 3 and 4 of cell 6 with the system assembled earlier in Figure 7 without applying extra forces. The other lines are matched automatically, due to the symmetry of the map along the $X$ and $Y$

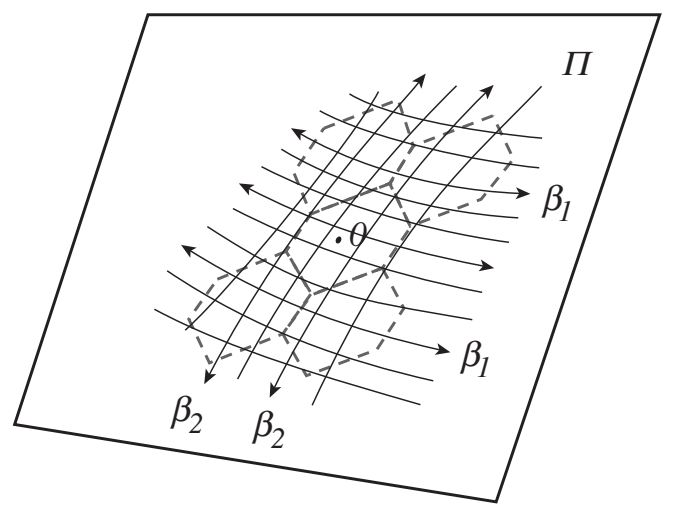

Figure 7. Section of the continuum shell. 


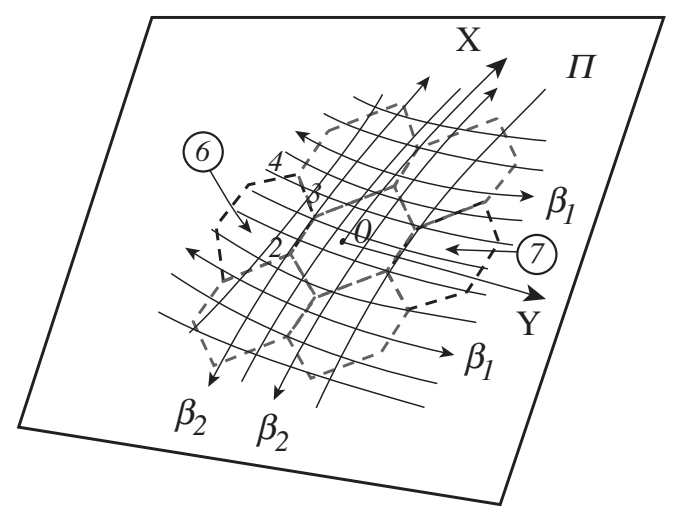

Figure 8. Local map of the shell.

axes. In other words, the distance between points $A$ and $B$ (with coordinates $\left(\alpha_{1 A}, \alpha_{2 A}\right)$ and $\left(\alpha_{1 B}, \alpha_{2 B}\right)$, respectively) within the small map $\Pi$ (Figure 8 ) is defined by the opening of only one angle $\Psi$.

Homogenization of the discrete system is performed in two steps. First, the free deformation of one cell and the small map $\Pi$ (that is, the deformation that does not have any external limitations) are considered. Second, the cells are joined into the continuum net using a specific rule.

For the first step, we consider that the sharp angle $\alpha_{0}$ is close to a straight angle. The assumption is that angle $\alpha_{0} \neq \pi / 2$, and the square of the cosine of this angle, $\alpha=\cos \alpha_{0}$, can be neglected in the calculations below.

Let us identify the projections of the unit length vectors:

$$
\begin{gathered}
\vec{e}_{4}=\{0,0,1\}, \quad \vec{e}_{1}=\{1,0,0\}, \quad \vec{e}_{2}=\left\{-a_{1} \alpha, a_{2} \alpha, a_{3}\right\}, \quad \vec{e}=\left\{-\psi_{1}, \psi_{2},-\alpha \psi_{3}\right\}, \\
\vec{e}^{\prime}=\left\{-\psi_{1},-\psi_{2},-\alpha \psi_{3}\right\}, \quad \text { and } \quad \vec{e}_{3}=\left\{-\alpha a_{1}^{\prime}, 0, a_{3}^{\prime}\right\} .
\end{gathered}
$$

Considering the relations

$$
\vec{e}_{2} \cdot \vec{e}=-\alpha, \quad \vec{e}_{1} \cdot \vec{e}_{2}=-\alpha, \quad \vec{e}_{3} \cdot \vec{e}=\alpha, \quad \vec{e}_{1}^{2}=\vec{e}^{2}=\vec{e}_{2}^{2}=\vec{e}_{3}^{2}=1,
$$

and the fact that vectors $\vec{e}, \vec{e}_{2}$, and $\vec{e}_{3}$ are located on the same plane, or

$$
\left(\vec{e}_{2} \times \vec{e}\right) \cdot \vec{e}_{3}=0
$$

we have

$$
\begin{aligned}
& a_{1}=1, \quad-a_{1} \psi_{1}+a_{2} \psi_{2}-a_{3} \psi_{3}=1, \quad \psi_{1} a_{1}^{\prime}-\psi_{3} a_{3}^{\prime}=1, \\
& \underline{\alpha^{2} \psi_{3}^{2}}+\psi_{1}^{2}+\psi_{2}^{2}=1, \quad \underline{a_{1}^{2} \alpha^{2}+a_{2}^{2} \alpha^{2}}+a_{3}^{2}=1, \quad \underline{\alpha^{2} a_{1}^{\prime 2}}+a_{3}^{\prime 2}=1, \\
& \underline{a_{2} \psi_{3} \alpha^{2}}+a_{3} a_{1}^{\prime} \psi_{2}+a_{3}^{\prime} a_{1} \psi_{2}+a_{3}^{\prime} a_{2} \psi_{1}=0 .
\end{aligned}
$$

There are eight unknowns in the seven equations. If all the unknowns are expressed through one parameter $\psi_{1}$, and $\alpha$ is a small number that allows the underlined elements in (4) to be omitted, the 
result is

$$
\begin{gathered}
a_{1}=1, \quad a_{3}^{2}=a_{3}^{\prime 2}=1, \quad \psi_{1}^{2}+\psi_{2}^{2}=1, \\
\psi_{3}=-\psi_{1}^{3}+\psi_{1}^{2}-\psi_{2}^{2} \psi_{1}+\psi_{2}^{2}, \quad a_{1}^{\prime}=\frac{1-\psi_{3}}{\psi_{1}}, \quad a_{2}=\frac{1-\psi_{3}-\psi_{1}}{\psi_{2}} .
\end{gathered}
$$

The angle between the $Z$ axis and vector $\left(\vec{e}+\vec{e}^{\prime}\right)$ is designated by $\lambda$, while the angle between surfaces 2 and 5 is expressed as $2 \beta$ (Figure 4). According to the designations introduced earlier, we obtain

$$
\lambda=\alpha \psi_{3} \quad \text { and } \quad \beta=\alpha a_{2} .
$$

The length of the line between the centers of sides 2 and 5 equals $2 b \psi_{2}$, while the length between points 1 and 4 (measured along the middle line of the trapezoid) equals $b\left(1+2 \psi_{1}\right)$. Therefore, the principal curvature radii can be grouped with the symmetrical free cell (and with the small map $\Pi$ ) by

$$
\rho_{1}=\frac{b\left(1+2 \psi_{1}\right)}{2 \alpha \psi_{3}} \quad \text { and } \quad \rho_{2}=\frac{b \psi_{2}}{\alpha a_{2}} .
$$

When $\psi_{1} \rightarrow 1$, the values of $a_{2}$ and $\psi_{3}$ approach zero; furthermore, the curvature radii at the location where the "folded" shape of the cell meets are equal to infinity.

When the coordinate $\alpha_{1}$ is changed by $b$ within the small map $\Pi$ (Figure 8) without connecting the incorporated cells, the distance along the line $\beta_{1}$ on the tangent plane $\Pi$ is equal to $b\left(1+\psi_{1}\right) / 2$; when the coordinate $\alpha_{2}$ is changed by $b$, the segment is equal to $b \psi_{2}$.

Therefore, when the cells are only in contact along the middles lines of the osculating trapezoids close to point $O$ (including the small number of free cells that are inside map $\Pi$ ), the first and the second quadratic forms of the surface can be calculated using

$$
\begin{gathered}
d s^{2}=A_{1}^{2} d \alpha_{1}^{2}+A_{2}^{2} d \alpha_{2}^{2}, \\
A_{1}=\left(1+\psi_{1}\right) / 2, \quad A_{2}=\psi_{2}, \\
B\left(d \alpha_{1}, d \alpha_{2}\right)=\frac{A_{1}^{2} d \alpha_{1}^{2}}{\rho_{1}}+\frac{A_{2}^{2} d \alpha_{2}^{2}}{\rho_{2}} .
\end{gathered}
$$

The written correlations define the connectivity described with the quadratic forms $\omega^{i}$ and $\omega_{i}^{j}$ [Cartan 1945] that are linear against the differentials $d \vec{\beta}_{1}$ and $d \vec{\beta}_{2}$. In addition,

$$
d \vec{r}=\omega^{i} \vec{\beta}_{i} \quad \text { and } \quad d \vec{\beta}_{i}=\omega_{i}^{j} \vec{\beta}_{j}
$$

Equation (9) and the subsequent formulas use a tensor notation and contracted repetitive indices, where $d \vec{r}$ means a vector of destination between the points on surface $\Pi$ that differ by the Lagrangian coordinates of $\left(d \alpha_{1}, d \alpha_{2}\right)$.

In the second step of homogenization, the basic assumption describing the global transformation of the net containing mirror-symmetrical six-link loops is made. The cells connected according to the sketch in Figure 2 deploy into a shell in such a way that the connectivity (expressed by (9)) is locally converted, with both surfaces $\Pi$ and $\Pi_{1}$ being smooth manifolds. This is performed by rigidly displacing the small free segment of the tangent plane from initial position $\Pi$ (the fragment of which is shown in Figure 9) to position $\Pi_{1}$, which is shown on the left side of the same picture. This requirement ensures 


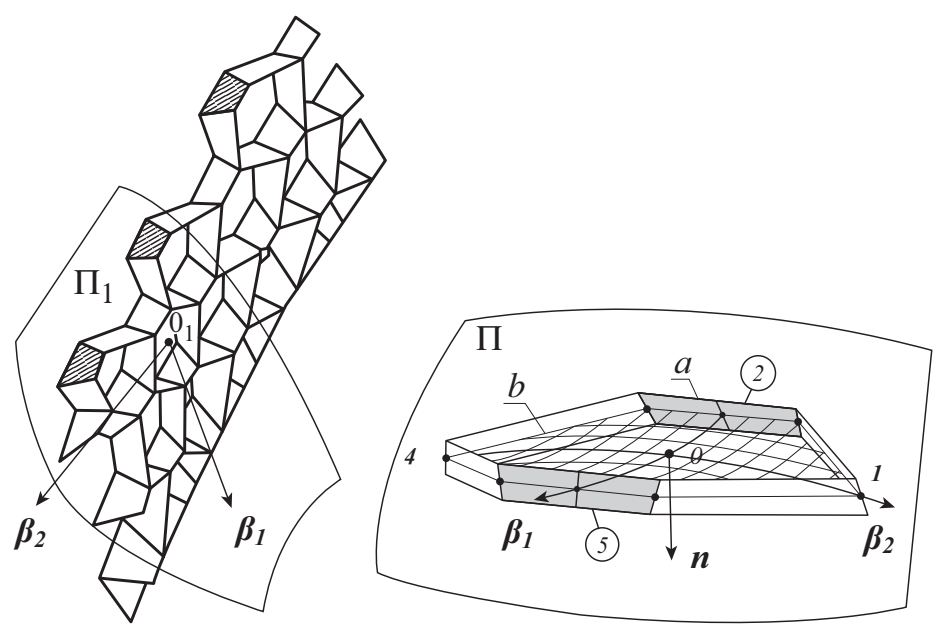

Figure 9. Movement of the local map in space.

the deformation of the shell without stretching the middle surface. Only those plates that connect the strips can be twisted.

Because the thickness of any plate is insignificant compared to its length and width, the potential energy of the elastic deformation of the shell is minimal; the deployment method described above would therefore require less extra energy than any other deployment technique.

To specify the forms of $\omega^{i}$ and $\omega_{i}^{j}$ relative to surface $\Pi_{1}$, it is sufficient to convert vectors $d \vec{\alpha}_{i}, \vec{\alpha}_{i}$, and $d \vec{r}$ by making them consistent with the Euler angles that appear after aligning points $O$ and $O_{1}$. The converted forms of $\omega^{i}$ and $\omega_{i}^{j}$ should satisfy the three Gauss-Codazzi relations, which are equations ensuring the system integrability condition (9) [Novozhilov 1962].

Due to the linearity of the forms (9) against $d \vec{\beta}_{1}, d \vec{\beta}_{2}, \vec{\beta}_{1}$, and $\vec{\beta}_{2}$ and the basic assumption on deformation of the net described above, the Euler angles would not be a part of the Gauss-Codazzi equations. Therefore, the resolved system of three equations would only have one variable function $\Psi$ and would become overdetermined. To ensure the integrability of the Gauss-Codazzi equations without changing the symmetry of the cells and the metrics of the shell, plate-twisting should be considered.

Equations (5) and (8) can be expressed in terms of plate-twisting as

$$
b_{11}=\frac{A_{1}^{2}\left(2 a \psi_{3}-\phi_{11}\right)}{b\left(1+2 \psi_{1}\right)}, \quad b_{12}=0, \quad \text { and } \quad b_{22}=\frac{A_{2}^{2}\left(\alpha a_{2}-\phi_{22}\right)}{b \psi_{2}},
$$

where $b_{11}, b_{12}$, and $b_{22}$ are coefficients of the second quadratic form of surface $B\left(d \alpha_{1}, d \alpha_{2}\right)=b_{i j} d \alpha_{i} d \alpha_{j}$ (indices $i$ and $j$ are 1 and 2, respectively); the functions $\phi_{11}$ and $\phi_{22}$ will be defined. The coefficient $b_{12}$ is considered equal to zero because the curvature lines in symmetrical cells are always orthogonal.

The coefficients of the first and second quadratic forms from (6) and (10) depend on three functions $\left(\phi_{11}, \phi_{22}\right.$, and $\left.\Psi\right)$, and they need to meet the criteria of the three Gauss-Codazzi compatibility equations

$$
\frac{\partial}{\partial \alpha_{2}}\left(\frac{\partial A_{1}}{A_{2} \partial \alpha_{2}}\right)+\frac{\partial}{\partial \alpha_{1}}\left(\frac{\partial A_{2}}{A_{1} \partial \alpha_{1}}\right)=-\frac{A_{1} A_{2}}{\rho_{1} \rho_{2}}, \quad \frac{\partial}{\partial \alpha_{2}}\left(\frac{\partial A_{1}}{\rho_{1}}\right)=\frac{\partial A_{1}}{\rho_{2} \partial \alpha_{2}}, \quad \frac{\partial}{\partial \alpha_{1}}\left(\frac{A_{2}}{\rho_{2}}\right)=\frac{\partial A_{2}}{\rho_{1} \partial \alpha_{1}} .
$$


Therefore, (5)-(8), (10), and (11) enable the shape of the surface to be uniquely defined (continuously approximating the location of the trapezoid middle line in space) when the coordinates of the shell reference contour are known. Basically, three differential equations (11) for the three functions $\psi_{1}, \phi_{11}$, and $\phi_{22}$ define the field (smooth manifold) in the coordinates $\alpha_{1}$ and $\alpha_{2}$, spread in three-dimensional space. The solution to (11) makes it possible to study the kinematics of the lattice shells without prior stress analysis of small plates.

\section{Application to specific problems}

Let us apply general correlations to the rotational shells. Equation (11) is simplified, and the derivatives with respect to argument $\alpha_{2}$ disappear; nevertheless, $\phi_{11}=0$. Therefore, two equations remain, and $\rho_{2}$ can be determined from the first equation:

$$
\frac{1}{\rho_{2}}=-\frac{\rho_{1}}{A_{1} A_{2}} \frac{\partial}{\partial \alpha_{1}}\left(\frac{\partial A_{2}}{A_{1} \partial \alpha_{1}}\right)
$$

If this expression is substituted into the second equation in (11), we have an ordinary differential equation of the second order for the function $\psi_{0}=\partial \psi_{2} / \partial \alpha_{1}$ :

$$
\frac{\partial}{\partial \alpha_{1}}\left(\frac{\rho_{1} \partial}{A_{1} \partial \alpha_{1}}\left(\frac{\psi_{0}}{A_{1}}\right)\right)=-\frac{\psi_{0}}{\rho_{1}} .
$$

Once $\psi_{2}$ is determined from this equation (up to two constants), the twisting parameter for the plate $\phi_{22}$ can be calculated only based on (11) and (12).

Knowing the function $\psi_{2}\left(\alpha_{1}\right)$, we can find the coordinates of the shell surface. In this equation, the closed shell is a finite-dimensional mechanism with the geometry dependent on the opening of only two cells located on the curvatures of $D_{1}$ and $D_{2}$ (Figure 10). It is also important to address the structure (13), which is close to the differential equation of second order with a large coefficient on the top derivative, and a rotational curvature that is close to the chain line approximating a straight line when the $\alpha$ angle is small.

The maximum opening of the cell is limited, and the closed rotational shell has the following interesting feature (Figure 10). If the shell is turned over with its cells open to the limit on the bottom, and the "bases" $D_{1}$ and $D_{2}$ are switched, the shell has to take the original shape because of its gravity and the lack of friction on base $D_{2}$.
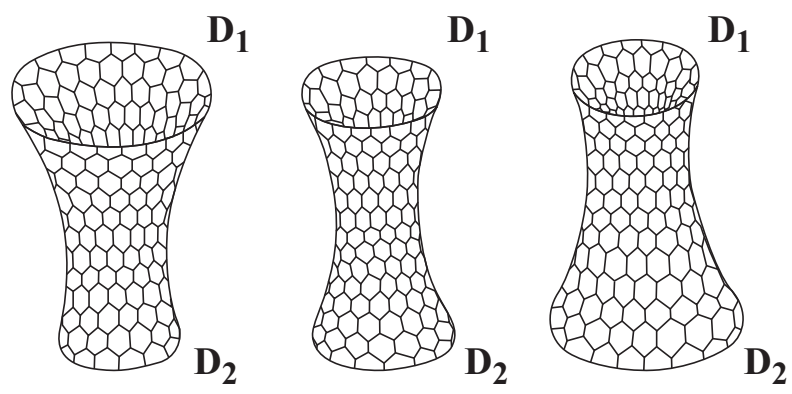

Figure 10. Change in shape of the hyperbolic shell under gravitation. 
As (5) shows, the honeycomb shells could increase the curvature during the deployment of simply connected packages in Figure 2, much like a crayfish squeezing a victim with its claw within the volume $V$ (Figure 11).

The simply connected honeycomb shell can occupy an extremely small volume. If $\psi_{2}=0$, then $A_{2}=0$, and the area of the shell is zero. This situation is possible in two cases: when $\Psi=0$ or when $\Psi=2 \pi$. The first option corresponds to the initial position of the package in Figure 1, while the second option corresponds to a wrapped "crib sheet" (or "cheat sheet"). The process of the shell "collapsing" into a point is shown in Figure 12 for one strip. The picture would not change if one strip was replaced with the simply connected lattice shell.

The stated features can serve as the basis for an advanced transportation technology of lattice systems: the wrapped strips are packed into a small box with dimensions similar to $(a+b)$ and transported at a long distance; the strips can subsequently be connected according to Figure 1 and unwrapped into the designed position.

The model shown in Figures 13-15 demonstrates the abovementioned features.

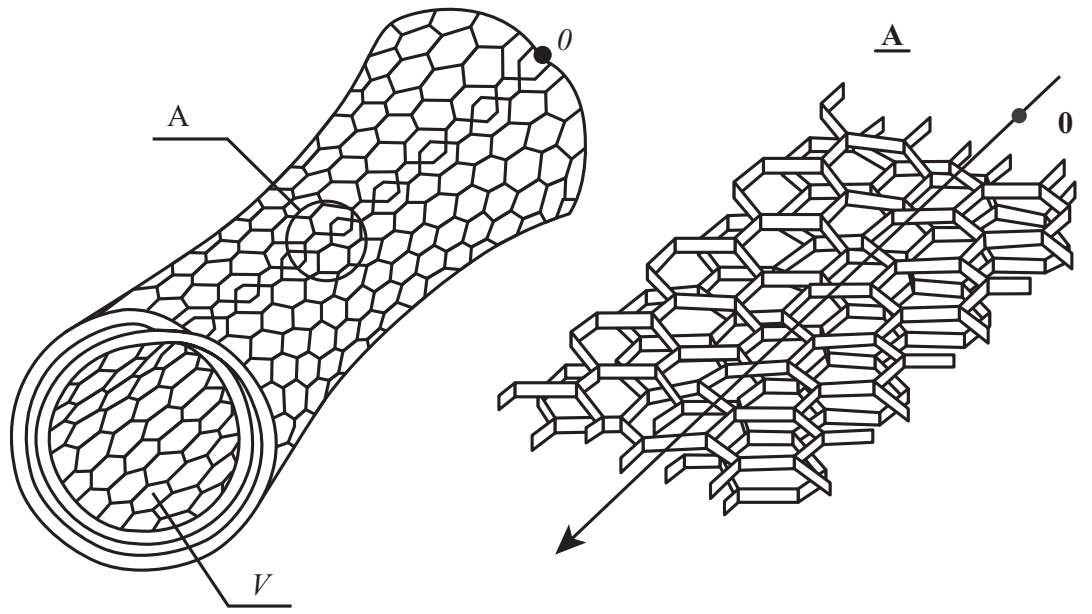

Figure 11. Reduction in internal volume when the shell is deployed. On the right, the arrow indicates the intersection line arising at deployment. The volume $V$ within the surface reduces when the shell deploys.

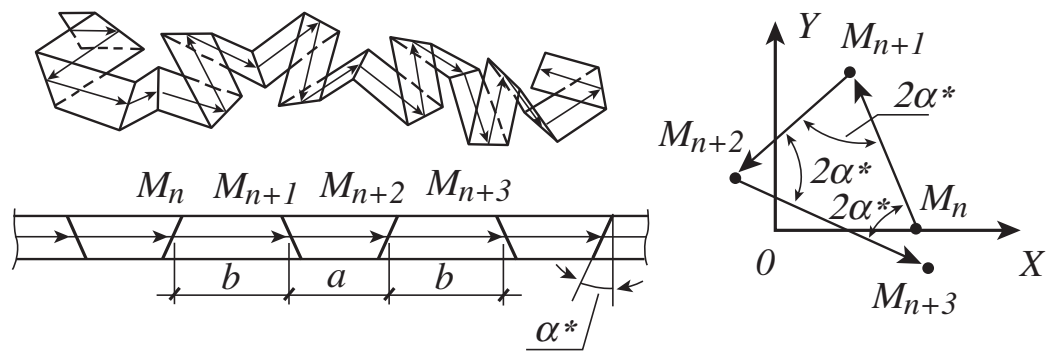

Figure 12. Strip packaged as a "crib sheet". 


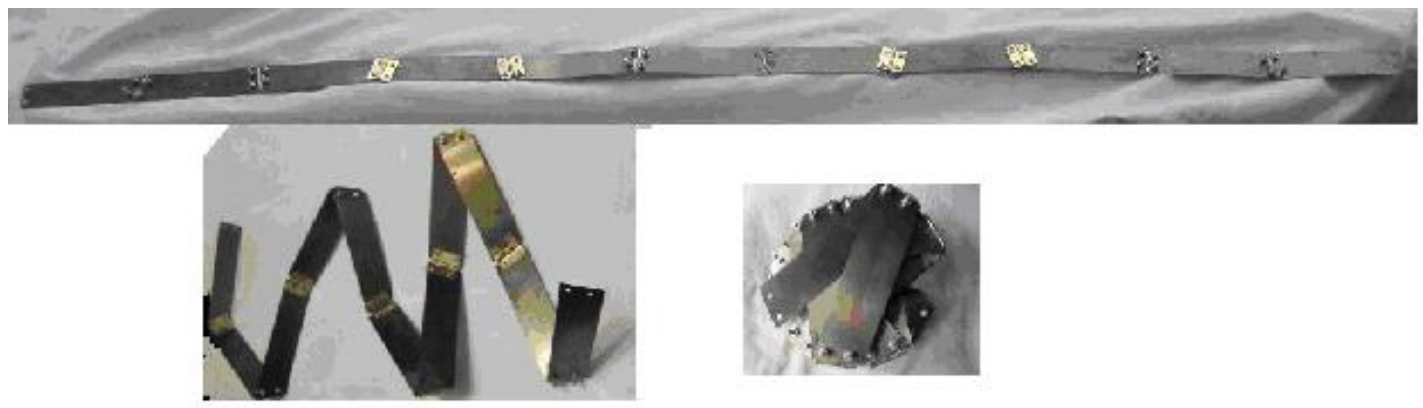

Figure 13. Strip in initial, intermediate, and packed states.

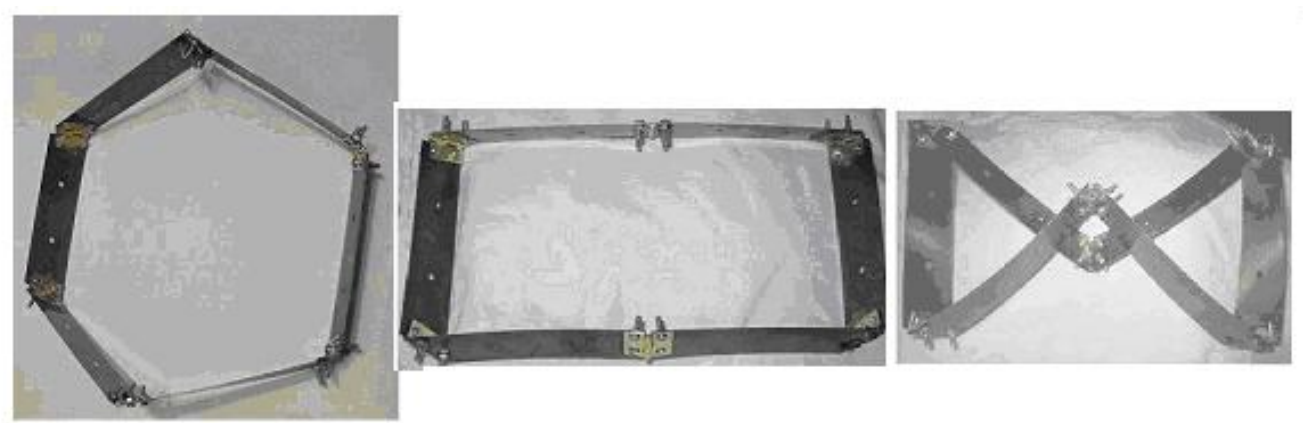

Figure 14. Cell at different $\Psi$ angles.

Figures 13 and 14 show a strip and a cell at the stage of preliminary assembly. The strips are put together from $0.2 \mathrm{~mm}$-thick plates (the trapezoid middle line is $115 \mathrm{~mm}$ long and $30 \mathrm{~mm}$ high, the sharp angle at the base is $75^{\circ}$ ) that are made of high-carbon steel with a yield strength of $1000 \mathrm{MPa}$. The hinged loops, which are $0.8 \mathrm{~mm}$ thick, are made of mild steel.

The loops and the plates are glued at $170^{\circ} \mathrm{C}$ with a special film adhesive after preliminary assembly in the casing ensuring $0.1 \mathrm{~mm}$ accuracy of linear dimensions.

Figure 13 shows the consistent process of packing the strip into a coil-globule that looks like a school crib.

Figure 14 demonstrates the deformation of the mirror-symmetric six-link loop when the dihedral angle $\Psi$ is changed. When $\Psi=2 \pi$, the cell's area is maximal. Further increase in this angle results in collapse of the cell (and the entire shell) because the area of any simply connected section of the lattice surface becomes negligibly small.

Figure 15, left, shows a model of the lattice shell comprising twenty strips joined into a single system with diameter $3 \mathrm{~mm}$ nylon thermoplastic rivets. The contacting surfaces are put together with six rivets: by two via hinged loops and by two along the middle line. The boundary conditions on the contours of the rotational shells are met by rigid rings where the hinged loops are attached.

If there were quite a few strips, then the arbitrarily set boundary conditions (diameters of the top and bottom rings) could change over a wide range and the lattice shell could simulate the situation represented 

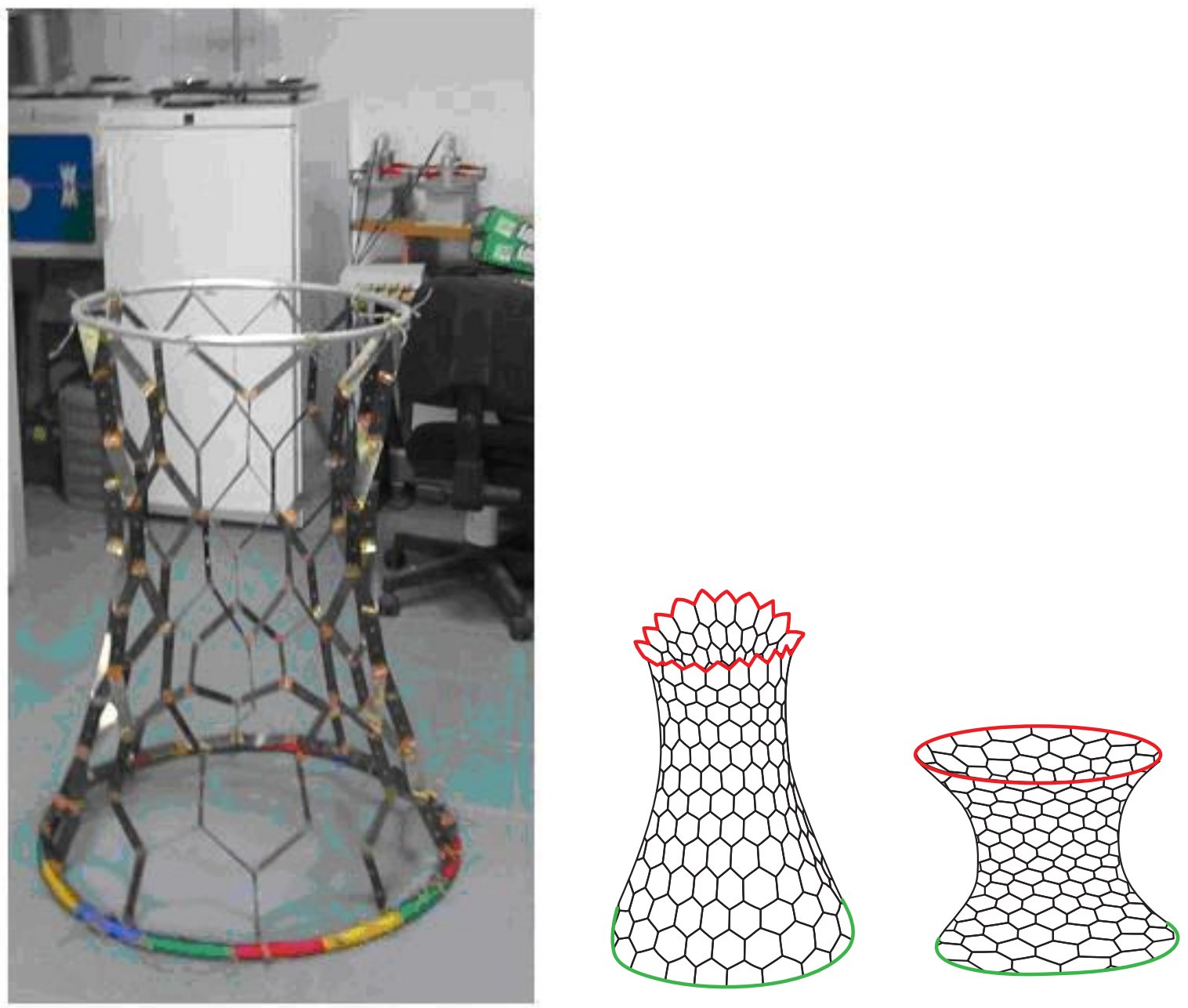

Figure 15. Left: model of the lattice shell. Right: Shell with a variable surface.

in Figure 10. If the cylinder was cut along the generating line, the crayfish behavior shown in Figure 11 would be reproduced.

If the rigid top ring was replaced with a flexible hose, as shown in red in Figure 15, right, then by injecting air inside this chamber, we would create a surface of variable area simulating a piston-free press or a pump. Obviously, such a surface can be used as a kagome structure upside down (a basketball hoop) with very few degrees of freedom. It would be much more difficult for players to shoot a ball through this basket than in a normal basketball game.

Figures 10-15 do not encompass all of the possible thin wall six-link loop structures [Hutchinson et al. 2003; Grachev and Neustadt 2008; 2009].

It should be emphasized once again that the deployable systems described above are not classic mechanisms with absolute rigid links. Their deployment is possible only if the plates are thin and durable, which explains why the proposed mechanisms based on the principles of mobility of the Bricard and 
Schatz six-link loops [Bricard 1927; Schatz 1975; Phillips 1984] have not been widely applied. Materials developed in recent decades, including shape memory alloys, encourage the development of new mechanisms of deployable systems and new applications in such fields as traditional engineering, medicine, and biotechnology.

\section{Acknowledgements}

The authors express their cordial thanks to the staff members of Samara Aerospace University, V. A. Koshelev and V. D. Falkin, and representatives of the Laktest laboratory, G. I. Weingarten and G. A. Makarov, for their tremendous support in the manufacturing of the lattice shells.

\section{References}

[Bricard 1927] R. Bricard, Leçons de cinématique, 2: Cinématique appliquée, Gauthier-Villars, Paris, 1927.

[Cartan 1945] É. Cartan, Les systèmes différentiels extérieurs et leurs applications géométriques, Actualités Sci. Ind. 994, Hermann, Paris, 1945.

[Chen and You 2008a] Y. Chen and Z. You, "An extended Myard linkage and its derived $6 R$ linkage", J. Mech. Des. (ASME) 130:5 (2008), Article ID \#052301.

[Chen and You 2008b] Y. Chen and Z. You, "On mobile assemblies of Bennett linkages", Proc. R. Soc. Lond. A 464:2093 (2008), 1275-1283.

[Gan and Pellegrino 2006] W. Gan and S. Pellegrino, "Numerical approach to the kinematic analysis of deployable structures forming a closed loop", Proc. Inst. Mech. Eng. C, J. Mech. Eng. Sci. 220:7 (2006), 1045-1056.

[Gantes 2001] C. J. Gantes, Deployable structures: analysis and design, WIT Press, Boston, 2001.

[Grachev and Neustadt 1995] V. A. Grachev and Y. S. Neustadt, Континуальные трансформирующиеся оболочки из прямолинейных полос (Continuum deployable shells made of rectilinear strips), Tertsia, St. Petersburg, 1995.

[Grachev and Neustadt 1996] V. A. Grachev and Y. S. Neustadt, Управляемые трансформирующиеся оболочки (Controlled deployable shells), St. Petersburg University Press, St. Petersburg, 1996.

[Grachev and Neustadt 1999a] V. A. Grachev and Y. S. Neustadt, “Способ изготовления плоских и пространственных сотовых структур и конструкций на их основе (Manufacturing process for plain and spatial honeycomb structures and constructions based on them)", 1999, http://ru-patent.info/21/25-29/2126875.html. Russian patent 2126875, filed 15 June 1992.

[Grachev and Neustadt 1999b] V. A. Grachev and Y. S. Neustadt, Трансформирующиеся среды из прямолинейных полос (Deployable media made of rectilinear strips), St. Petersburg University Press, St. Petersburg, 1999.

[Grachev and Neustadt 2008] V. A. Grachev and Y. S. Neustadt, “Трансформирующиеся системы на основе правильных шестизвенников (Deployable systems based on regular six link loops)”, pp. 131-139 in Математика, компьютер, образование, 2.5: Вычислительные методы и математическое моделирование (Mathematics, computer, education, 2.5: Calculation methods and mathematical modeling) (Dubna, 2008), edited by G. Y. Riznichenko, Scientific Publishing Center "Regular and Chaotic Dynamics", Izhevsk, 2008.

[Hutchinson et al. 2003] R. G. Hutchinson, N. Wicks, A. G. Evans, N. A. Fleck, and J. W. Hutchinson, "Kagome plate structures for actuation", Int. J. Solids Struct. 40:25 (2003), 6969-6980.

[Jensen and Pellegrino 2002] F. Jensen and S. Pellegrino, "Expandable structures formed by hinged plates", pp. 263-272 in Space structures 5 (Surrey, 2002), vol. 1, edited by G. A. R. Parke and P. Disney, Thomas Telford, London, 2002.

[Leung and Guest 2007] A. C. H. Leung and S. D. Guest, "Single member actuation of kagome lattice structures", J. Mech. Mater. Struct. 2:2 (2007), 303-317.

[Luchsinger et al. 2004] R. H. Luchsinger, M. Pedretti, and A. Reinhard, "Pressure induced stability: from pneumatic structures to Tensairity®", J. Bionic Eng. 1:3 (2004), 141-148.

[Lurye 1961] A. I. Lurye, Аналитическая механика (Analytical mechanics), GIFML, Moscow, 1961. 
[Novozhilov 1962] V. V. Novozhilov, Теория тонких оболочек (The theory of thin shells), Sudpromgiz, Leningrad, 1962. Reprinted by St. Petersburg University Press, St. Petersburg, 2010.

[Otto and Rasch 1995] F. Otto and B. Rasch, Finding form: towards an architecture of the minimal, Axel Menges, Stuttgart, 1995.

[Phillips 1984] J. Phillips, Freedom in machinery, 2: Screw theory exemplified, Cambridge University Press, Cambridge, 1984.

[Schatz 1975] P. Schatz, Rhythmusforschung und Technik, Freiesleben, Stuttgart, 1975.

[Sclater and Chironis 2011] N. Sclater and N. P. Chironis, Mechanisms and mechanical devices sourcebook, 5th ed., McGraw Hill, New York, 2011.

[Swank 1991] M. W. Swank, "Non-planar expandable honeycomb structure", 1991, http://www.google.com/patents/US4981744. USA patent 4981744, filed 24 April 1990.

[Vepa 2010] R. Vepa, Dynamics of smart structures, Wiley, Hoboken, NJ, 2010.

Received 4 Sep 2012. Revised 7 Nov 2012. Accepted 18 Nov 2012.

VLADIMIR A. GRACHEV: grach_va@rambler.ru

Samara State Architectural and Building University, 194 Molodogvardeyskaya Str., Samara, 443001, Russia

YURIY S. NEUSTADT: neustadt99@mail.ru

Samara State Architectural and Building University, 194 Molodogvardeyskaya Str., Samara, 443001, Russia 


\title{
JOURNAL OF MECHANICS OF MATERIALS AND STRUCTURES
}

\author{
msp.org/jomms
}

\author{
Founded by Charles R. Steele and Marie-Louise Steele
}

EDITORIAL BOARD

ADAIR R. AGUIAR

KATIA BERTOLDI

University of São Paulo at São Carlos, Brazil

DAVIDE BIGONI

Harvard University, USA

IWONA JASIUK

University of Trento, Italy

Thomas J. PENCE

University of Illinois at Urbana-Champaign, USA

YASUHIDE SHINDO

Michigan State University, USA

DAVID STEIGMANN

Tohoku University, Japan

University of California at Berkeley

\section{ADVISORY BOARD}

J. P. CARTER University of Sydney, Australia

R. M. Christensen Stanford University, USA

G. M. L. GLADWELL University of Waterloo, Canada

D. H. Hodges Georgia Institute of Technology, USA

J. HUTCHINSON Harvard University, USA

C. HwU National Cheng Kung University, Taiwan

B. L. KARIHALOO University of Wales, UK

Y. Y. KIM Seoul National University, Republic of Korea

Z. Mroz Academy of Science, Poland

D. PAMPlonA Universidade Católica do Rio de Janeiro, Brazil

M. B. RubiN Technion, Haifa, Israel

A. N. SHUPIKOV Ukrainian Academy of Sciences, Ukraine

T. TARNAI University Budapest, Hungary

F. Y. M. WAN University of California, Irvine, USA

P. WRIGGERS Universität Hannover, Germany

W. YANG Tsinghua University, China

F. ZIEGLER Technische Universität Wien, Austria

PRODUCTION production@msp.org

SILVIO LEVY Scientific Editor

Cover photo: Mando Gomez, www.mandolux.com

See msp.org/jomms for submission guidelines.

JoMMS (ISSN 1559-3959) at Mathematical Sciences Publishers, 798 Evans Hall \#6840, c/o University of California, Berkeley, CA 94720-3840, is published in 10 issues a year. The subscription price for 2013 is US \$555/year for the electronic version, and $\$ 705 /$ year (+\$60, if shipping outside the US) for print and electronic. Subscriptions, requests for back issues, and changes of address should be sent to MSP.

JoMMS peer-review and production is managed by EditFLOW ${ }^{\circledR}$ from Mathematical Sciences Publishers.

PUBLISHED BY

mathematical sciences publishers

nonprofit scientific publishing

http://msp.org/

(C) 2013 Mathematical Sciences Publishers 


\section{Journal of Mechanics of Materials and Structures}

\section{Volume 8, No.:2-4 April-June 2013}

A diffuse cohesive energy approach to fracture and plasticity: the one-dimensional case

Gianpietro Del Piero, Giovanni Lancioni and Riccardo March 109

Continuum deployable shells made of thin plates

Vladimir A. GracheV and Yuriy S. NeUSTAdT

Self-folding of a slender microbeam and thin film: an elastica model

JIANLIN LIU and JUNG HoON LEE 169

Dispersion of guided waves in initially stressed layered plates

JIANGONG YU and SHENLEI LI 185

Empirical models for predicting protective properties of concrete shields against

high-speed impact

GABI BEN-Dor, ANATOLY DUBinsky and Tov ELPERIN

Transient response of a general anisotropic solid to dislocation growth: alternative formulation LOUIS MILTON BROCK 